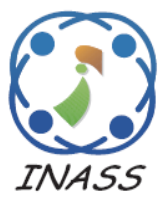

\title{
A Sparse Representation of Social Media, Internet Query, and Surveillance Data to Forecast Dengue Case Number using Hybrid Decomposition- Bidirectional LSTM
}

\author{
Wiwik Anggraeni ${ }^{1,2}$ \\ Eko Mulyanto Yuniarno ${ }^{3}$ \\ Reza Fuad Rachmadi ${ }^{3}$ \\ Mauridhi Hery Purnomo ${ }^{1,3,5 *}$ \\ Pujiadi $^{4}$ \\ ${ }^{1}$ Department of Electrical Engineering, Institut Teknologi Sepuluh Nopember, Surabaya, Indonesia \\ ${ }^{2}$ Department of Information Systems, Institut Teknologi Sepuluh Nopember, Surabaya, Indonesia \\ ${ }^{3}$ Department of Computer Engineering, Institut Teknologi Sepuluh Nopember, Surabaya, Indonesia \\ ${ }^{4}$ Dengue Fever Eradication, Malang Regency Public Health Office, Malang, Indonesia \\ ${ }^{5}$ University Center of Excellence on Artificial Intelligence for Healthcare and Society (UCE AIHeS), Indonesia \\ * Corresponding author's Email: hery@ee.its.ac.id
}

\begin{abstract}
Dengue fever is an endemic disease that occurs throughout the year. Forecasting cases of dengue fever based on actual data is needed for monitoring and taking action. Recently, developing countries have faced problems related to the dengue fever surveillance system caused by the data delay factor. On the other hand, availability and access to health-related information on the internet have changed people's behaviors and habits. However, the effect of internet data usage has not been widely studied, especially in areas with different levels of internet penetration. This study examines the impact of dengue fever case reported data, Google Trends, Twitter, and climate data in areas with many cases and varying levels of internet penetration to forecast dengue fever cases. Split time-series cross-validation (STSCV) and blocked time-series cross-validation (BTSCV) are used to obtain various training and testing results. The hybrid Decomposition-Bidirectional Long Short-Term Memory(D-BiLSTM) method is proposed. D-BiLSTM applied to eight different scenarios across multiple level areas. According to the results of the experiments, the DBiLSTM model with STSCV outperforms the BTSCV. In the high internet penetration area, the average error is 9,517, while in the low internet penetration area, it is 5,188 . In areas with high internet penetration, adding the variables Google Trends and Twitter does not significantly reduce the error forecasting. However, in the low penetration area, the inclusion of Google Trends and Twitter significantly decreases errors. In general, the D-BiLSTM model performed well. Then, when compared with other approaches, the D-BiLSTM model as a whole can reduce the average RMSE and the average MAE of the comparison model by 94,120 and 45,132, respectively, in areas of high internet penetration with the best SMAPE model of 0.310. In the low internet penetration area, the average decline in RMSE and MAE was 54,390 and 19,362, with the best SMAPE model performance of 0.183.
\end{abstract}

Keywords: Dengue fever, Dynamic forecasting, Social media, Internet query, Decomposition, Bidirectional long short-term memory.

\section{Introduction}

Dengue Fever (DF) is a contagious disease caused by the dengue virus carried by the Aedes Aegypti mosquito [1]. These mosquitoes are found in tropical and subtropical areas, including Indonesia. DF is an endemic disease that occurs throughout the year, especially during the rainy season. This disease infects a large number of people in a short period [2]. In addition, DF is also the fastest spreading viral disease [3]. There are 50-100 million cases reported worldwide in 100 countries each year which cause 24,000 deaths. Approximately 2.5 billion people live in an endemic state of DF. Based on data compiled by WHO from 1968-2009, Indonesia was the country with the highest cases of DF in Southeast Asia [4]. DF has become one of Indonesia's major public 
health problems over the past 47 years as it causes rapid and multiple deaths [2]. DF cases in Indonesia increased significantly in 2019 [5], and many of them died. Delays in handling triggered this condition. One of the causes of delays in handling can be related to the data reporting system.

In several developing countries, including Indonesia, the provision of data quickly becomes a problem [6]. A robust, accurate, and reliable disease surveillance system for DF disease is currently unavailable [7, 8]. So far, the government has relied on hospital-based reporting, which is often left behind and sometimes causes delays in data availability [8], therefore requires revision [7]. The delay in reporting can create gaps in disease surveillance [6], leading to delays in disease detection and management [9]. It shows the need for alternative data sources that can describe cases of dengue fever in near real-time.

On the other hand, the current availability and accessibility of health-related information on the internet have altered how people use the internet [10, 11]. Although not everyone who searches for healthrelated terms is ill, there is a close relationship between the number of people who search for news about a particular illness and the number of people who have the symptoms. However, it can be an indication of the disease's spread [12]. Internet data, including social media has been widely used to monitor diseases such as influenza [13, 14], DF [6-8, 15-18], Zika [19], Chikungunya [9], Malaria [20], Lyme [21], and hand, foot, and mouth disease [22].

Although research has been carried out related to internet data or social media associated with DF, to the best of our knowledge, it has not yet been examined in detail about how different usage affects areas with different internet penetration rates. Most of the previous studies involved internet search data at the national level, whether used in predicting the number of DF cases [23] and DF outbreaks [24]. Previous studies only focused on the association between social media data and the number of reported cases and had not yet reached the predicted level $[6$, 15]. In addition, previous studies also involved data from related agencies and social media data only [6, $15,18]$. Otherwise, the number of DF cases is influenced by climatic factors such as rainfall [23], temperature [25], and humidity [26]. The use of climatic factors as a component in the forecasting process can also improve performance [27].

Various conditions and the results of the discussion of previous studies regarding the need to see the effect of usage in areas with different internet penetration rates and involving other factors in predicting DF cases are a challenge for the following study. Therefore, this study attempts to solve this challenge. This study aims to see how Google search data and social media influence forecasting DF cases number. This impact is investigated for areas with varying internet penetration rates by factoring in the real-time climate factor in each area. The climate factors involved include temperature, humidity, rainfall, and wind speed. In addition, this study also proposes a combination of dynamic time series and deep learning models to forecast the number of DF cases in several months ahead. This combination is called Decomposition-Bidirectional LSTM (DBiLSTM). This model is equipped with a crossvalidation process so that the model is more robust and has improved forecasting performance. Furthermore, this model is designed dynamic in terms of adding new input data.

Ultimately, the google query and Twitter results are expected to complement the surveillance data in providing information about future DF cases so that the health office can respond quickly in reducing morbidity and mortality. In addition, the optimal model obtained makes it possible to predict the number of DF events in real-time. This model will reduce DF's social costs and economic losses by eliminating the gap in reporting time in traditional surveillance systems. The remainder of this paper is organized as follows. Section 2 describes the previous related studies. In Section 3, the research areas, data sources, and methods are used. Section 4 discusses the results and discussion. Section 5 presented conclusions, as well as directions for future work.

\section{Related works}

\subsection{Involvement of internet query factors, social media, and climate in forecasting the DF cases number}

In recent years, digital footprints have become a potential source of data for health-related purposes. The digital tread is usable to explore disease patterns and health dynamics in a population. Wider internet penetration, increased use of mobile phones, and artificial intelligence in the field of digital epidemiology are promising approaches to assist disease surveillance systems caused by delays in data reporting [28]. This approach has the potential to address gaps in conventional surveillance systems, which often experience delays in reporting, underreporting, and a lack of supporting budgets because the costs involved are very high.

So far, there have been several studies involving internet search data. Most of these studies use Google 
Search data which is better known as Google Trends. These studies prove that Google Trends data correlates well with data on cases reported in the Health Agency, be it cases of DF [6, 8, 15, 16], Malaria [20], Zika [19], and hand, foot, and mouth [22], pertussis [29], and influenza [30]. Besides, Google Trends data is also stated to increase the accuracy of disease prediction, be it the prediction of the number of events $[13,17,19]$, population health behavior [18], and outbreaks [7]. Google Trends data can also indicate national epidemiological trends in annual and seasonal variations between years [16]. These studies declare the potential use of Google Trends data. It can be obtained more efficiently, faster, and at a lower cost than traditional reporting systems. Although Google Trends have been widely used in previous DF case research, according to our knowledge, still very rare analyzed how Google Trends influenced areas with different internet penetration rates. This study is relevant to what was suggested by $[8,9]$, which stated that the following research should analyze areas with different internet penetration rates. In addition, previous studies conducted analyzes at the national level. Dengue information at the national level is not ideal for making decisions locally [7]. The spatiotemporal policy is more suitable, especially for areas with high cases [9]. It is because information at the national level is aggregated from a heterogeneous spatial environment.

On the other hand, there are still few studies that report the potential use of Twitter data. This condition poses a challenge because the public interest in using Twitter is getting bigger. Besides, nowadays, Twitter has become one of the popular social media in Indonesia, besides Instagram and YouTube [31]. As of May 2020, Twitter users in Indonesia increased by $24 \%$ compared to 2019 [32]. Indonesia has become the fifth largest country for Twitter users after the UK and other significant countries [33]. In addition, it is also rare to combine the influence of climate variables and internet search data or social media. Most of the previous research used search query data or social media alone. Several previous studies stated that climatic factors primarily influenced cases of dengue fever. Human mobility, mosquito control, and temperature have different effects on the $[23,25,34]$. The trend in DF has a strong correlation with temperature and humidity. Besides that, it is also following the discussion presented by [18] that climate should detect DF cases. Likewise, climatic factors such as temperature, rainfall, humidity, and wind speed affect DF in Indonesia [35].

\subsection{The approach used in forecasting the DF cases number}

In a previous study, Google Trends data and Twitter data on DF were correlated using the Spearman correlation [15, 18] and Pearson correlation $[6,8,27]$. Some of them also involve a lag factor or delay effect $[6,18,27]$. Meanwhile, prior studies generally utilized the Autoregressive Likelihood Ratio [13], ARIMAX [17, 20], ARIMA $[16,20]$, SARIMA $[28,29]$, and SARIMA RuleBased [35] to estimate the number of cases and disease outbreaks. Furthermore, the Time Series Decomposition method [29] and the Autoregressive Model with Google Search [7] are also used. Besides, previous studies that employed social media data for case prediction and DF outbreaks often employed the Autoregressive Likelihood Ratio approach [13], ARIMAX [17], Autoregressive Model with Google Search [7]. Approaches in [7, 13, 17] are based on the classic time series approach.

This classic time series approach has some drawbacks and advantages. ARIMA and SARIMA are the most widely used forecast models. They perform well when the data is linear [36] and shortterm forecasting [22]. But that performance declines when used for medium- and long-term forecasting. In addition, it needs stationary tests in mean and variance over time, which takes a lot of time. ARIMA and SARIMA methods are forecasting methods that do not involve the influence between variables. In addition, ARIMA and SARIMA require us to find different parameters or degrees for other datasets, namely p, d, q [22, 29]. A combination of incorrect parameters will get bad results. Thus, the model's performance so a strong dependency on parameter setting ( $p d q)$. ARIMA and SARIMA have been developed by involving several other variables known as ARIMAX models and SARIMAX methods. Although it can affect other variables, the model is still difficult to overcome the non-linearity problem [36].

The classic Time series decomposition approach has the advantage of recognizing the trend, seasonal, cyclical, or random components in the data. However, this model cannot involve other variables such as ARIMA. Besides, Likelihood ratio autoregression is a model approach that can include more than one variable. It uses the likelihood ratio approach to the testing problems in threshold autoregression. This condition makes it difficult to choose the criteria bound. Therefore, it takes a strategy to address this threshold problem. The usual technique used is an estimation. 
The relationship between DF and climatic factors is complex, so the classical time series model is not easy to get a fit prediction result [36]. The deep learning approach offers more advantages in the health sector than traditional statistical models [37, 38 ] and is more frequently applied in predicting disease prevalence [39]. LSTM is often used for time series prediction. It has been successfully used to predict influenza trends and hand, foot, and mouth disease epidemics [40, 41]. LSTM was more widely used for data with large values in the previous study, including many disease events. However, LSTM is declared challenging to predict in areas with less incidence of disease [36] and is less accurate [40]. Research with location differentiation based on penetration in this study shows that the existing data is small and even zero, not stationary, and seasonal. Based on these facts, in this study, LSTM will be combined with the decomposition approach. It extracts time-series data that are often nonlinear and nonstationary without leaving the time domain [29]. So far, several decomposition-based hybrid models such as ANN-decomposition and SVMdecomposition have been developed to investigate time series in other cases. The findings show that the hybrid decomposition model will increase the original data's regularity and get more reliable forecasting results than the conventional model. Based on the advantages, disadvantages, and conditions of existing data that have been mentioned earlier, in this study, the Decomposition will be combined with a particular type of LSTM, namely Bidirectional LSTM, to produce forecasts with good performance. Then, to witness how robust the combination model of D-BiLSTM is, special crossvalidation will be carried out for time series data in this study. Several types of cross-validation are not all suitable for time series data [41].

\section{Materials and method}

\subsection{Study area}

This study uses two areas, each representing an urban area with a high internet penetration rate and an area where most areas can be categorized as rural with a lower internet level. These areas are the city of Surabaya and the Malang Regency.

Surabaya is the capital of the East Java province and Indonesia's second-largest city after Jakarta. Surabaya is also one of Southeast Asia's oldest port cities, with 160 sub-districts and a population density of 8,268 people per square kilometre. Surabaya City is located at $7^{\circ} 9^{\circ}-7^{\circ} 21^{\prime}$ South Latitude and $112^{\circ} 36^{\prime}$ $112^{\circ} 57^{\prime}$. Surabaya is mostly lowland, with an elevation of 3-6 meters above sea level [42].

Surabaya's Communication and Information Office announced that the city had won the Indonesia Digital Society Award for having the most digital community in Indonesia. Then, according to a survey conducted by the Indonesian Internet Service Providers Association from the second quarter of 2020 to the second quarter of 2021, Surabaya is a provincial capital with a high internet penetration rate $(83.0 \%)$, exceeding provincial and even national penetration rates of $73.7 \%$ [43].

Then, Malang regency is a plateau surrounded by several mountains and lowlands or valley areas at an altitude of 250-500 masl. Malang Regency is the second-largest district in East Java after Banyuwangi Regency and is the district with the largest population in East Java. Malang Regency has coordinates $112^{\circ}$ $17^{\prime}$ to $112^{\circ} 57^{\prime}$ East Longitude and $7^{\circ} 44^{\prime}$ to $8^{\circ} 26^{\prime}$ South Latitude. Malang Regency is also the thirdlargest district in Java Island. The total area is $3,530.65 \mathrm{~km} 2$ consisting of 378 villages with a population density of $831.33 / \mathrm{km}^{2}$ [44]. The level of internet penetration in the Malang Regency is $18.3 \%$.

\subsection{Dataset}

The data used in this study consisted of DF surveillance data, climate data including temperature, rainfall, humidity, and wind speed, Google Trends, and Twitter. The data period used is 2009-2019. The surveillance data were obtained from the Health Office in Malang and Surabaya Regencies. In contrast, the climate data were obtained from the Meteorology, Climatology, and Geophysics Agency of Karangploso and Juanda stations. Data from Google and Twitter is obtained by crawling data using several top keywords related to the term dengue fever commonly used by Indonesians, such as demam (in English fever), demam berdarah (in English dengue fever), dengue, dengue virus. dengue fever.

The data patterns and changes over time of each variable involved in the study are displayed in Fig. 1. The RCN variable represents the reported DF case number, TEMP represents temperature, and RH is relative humidity. Moreover, RF is rainfall, WDSP represents windspeed, TW is the number of tweets related to DF, and GT represents the number of Google Trends.

\subsection{Method}

This study analyses the correlation between data on Google Trends, Twitter, and climate affect the number of reported DF cases. Besides, this study also analyzes their influence on forecasting results. 

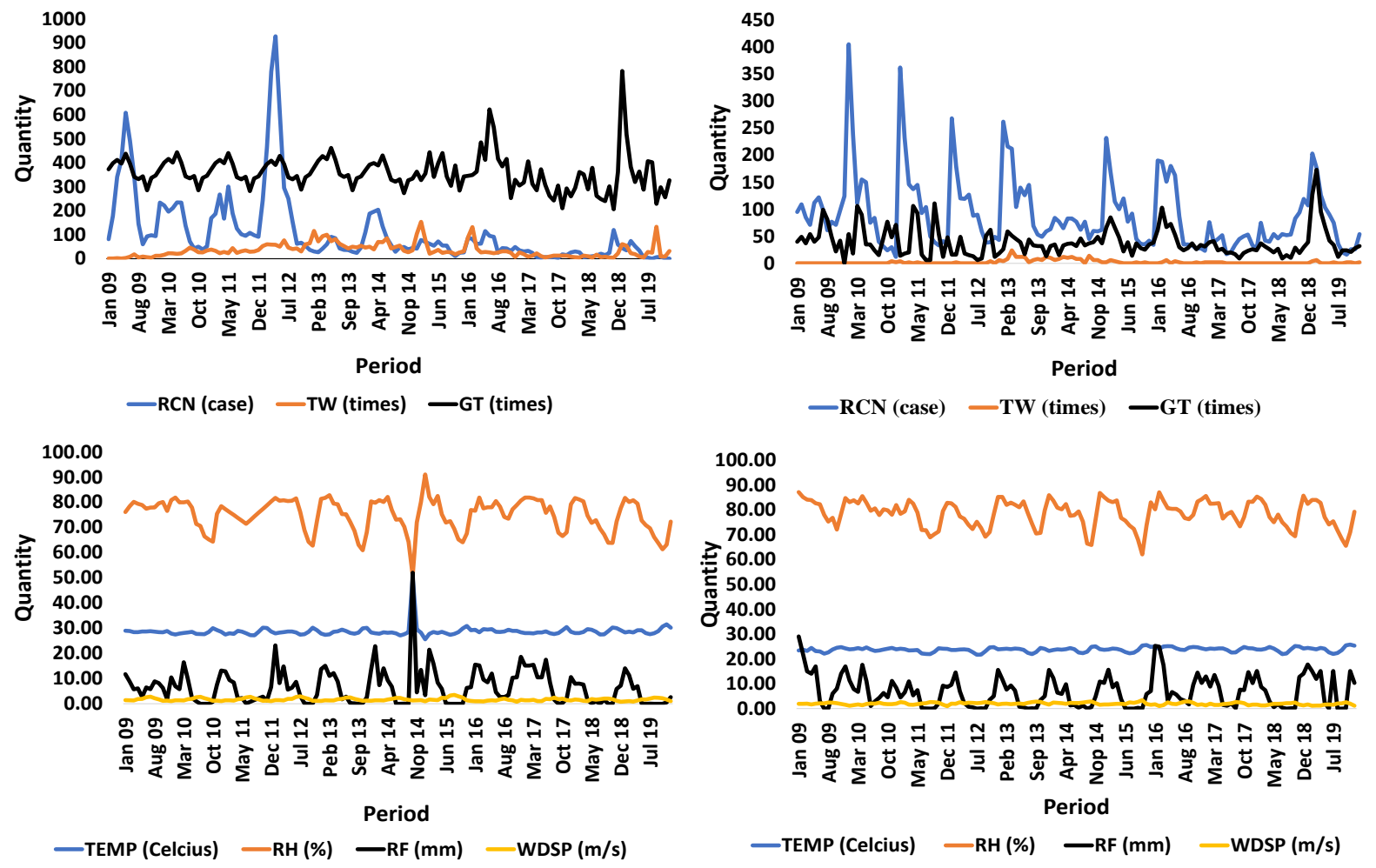

(a)

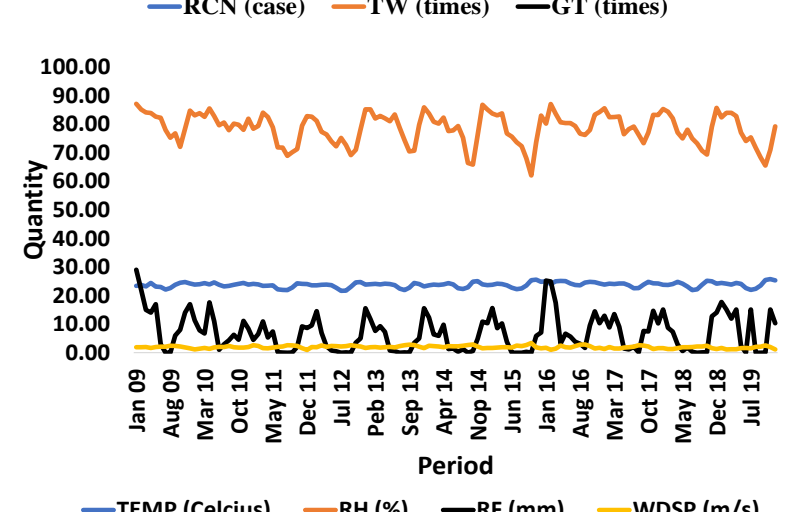

(b)

Figure. 1 Data plots of variables involved in each area: (a) Surabaya city, (b) Malang district.

This objective is obtained by building a DF surveillance forecasting model using climate data, including temperature, rainfall, humidity, wind speed, Google Trends, and Twitter. Modeling is carried out using a hybrid model, namely D-BiLSTM. The performance of this model has compared to other candidate models, such as ARIMAX [17], LASSO [34], SVM [37], and Neural Network (NN) [37]. The framework of this study is shown in Fig. 2.

\subsubsection{Correlation analysis}

How strong the relationship between the variables involved can be found using the correlation coefficient. The specific purpose of the correlation analysis in this study is to see how big the relationship between data on Google Trends, Twitter, and climate on the number of DF cases reported in each area. This study carried out the correlation with the Pearson product-moment $[6,8]$. The Pearson product-moment equation is presented in Eq. (1). The $r_{A, B}$ show the correlation coefficient for variables $A$ and $B$. Addition, $n$ the amount of data, $a_{t}$ and $b_{t}$ are the values of attributes $A$ and $B$ in the data $t$. While $\bar{A}, \bar{B}$ are the average of attribute $A$ and attribute $B$ values, as well as $\sigma_{A}, \sigma_{B}$ show the standard deviation of attribute $A$ and attribute $B$.

$$
r_{A, B}=\frac{\sum_{t=1}^{n}\left(a_{t}-\bar{A}\right)\left(b_{t}-\bar{B}\right)}{n \sigma_{A} \sigma_{B}}=\frac{\sum_{i=1}^{n}\left(a_{i}-b_{i}\right)-n \bar{A} \bar{B}}{n \sigma_{A} \sigma_{B}}
$$

\subsubsection{Decomposing the involved variables}

All data next are decomposed using Additive Decomposition. This decomposition produces three values for each variable, namely, trend, seasonality, and random components. In this study, time series decomposition uses the moving average (MA) method to examine trend cycles and seasonal behavior. The trend component $\left(T_{t}\right)$ is obtained using a $2 \times \mathrm{m}$-MA, while the de-trend is obtained by subtracting the DF $\left(Y_{t}\right)$ surveillance data trend. The seasonal component of each season is estimated by averaging the de-trend value in that season. The value of this seasonal component is then adjusted to ensure that it is close to zero. The seasonal variable can be calculated by stringing together these monthly values and then replicating the series for each year. As a result, the residue component $\left(S_{t}\right)$ is found by subtracting the seasonal components and the trend cycle. $R_{t}$ denotes the residue.

Mathematically, the Decomposition can be modeled like Eq. (2). Meanwhile, the additive model to capture seasonal variations in successive periods is presented in Eq. (3). The de-trend $\left(D_{t}\right)$ processes and the complete decomposition are given in Eq. (4) and Eq. (5) [29]. 


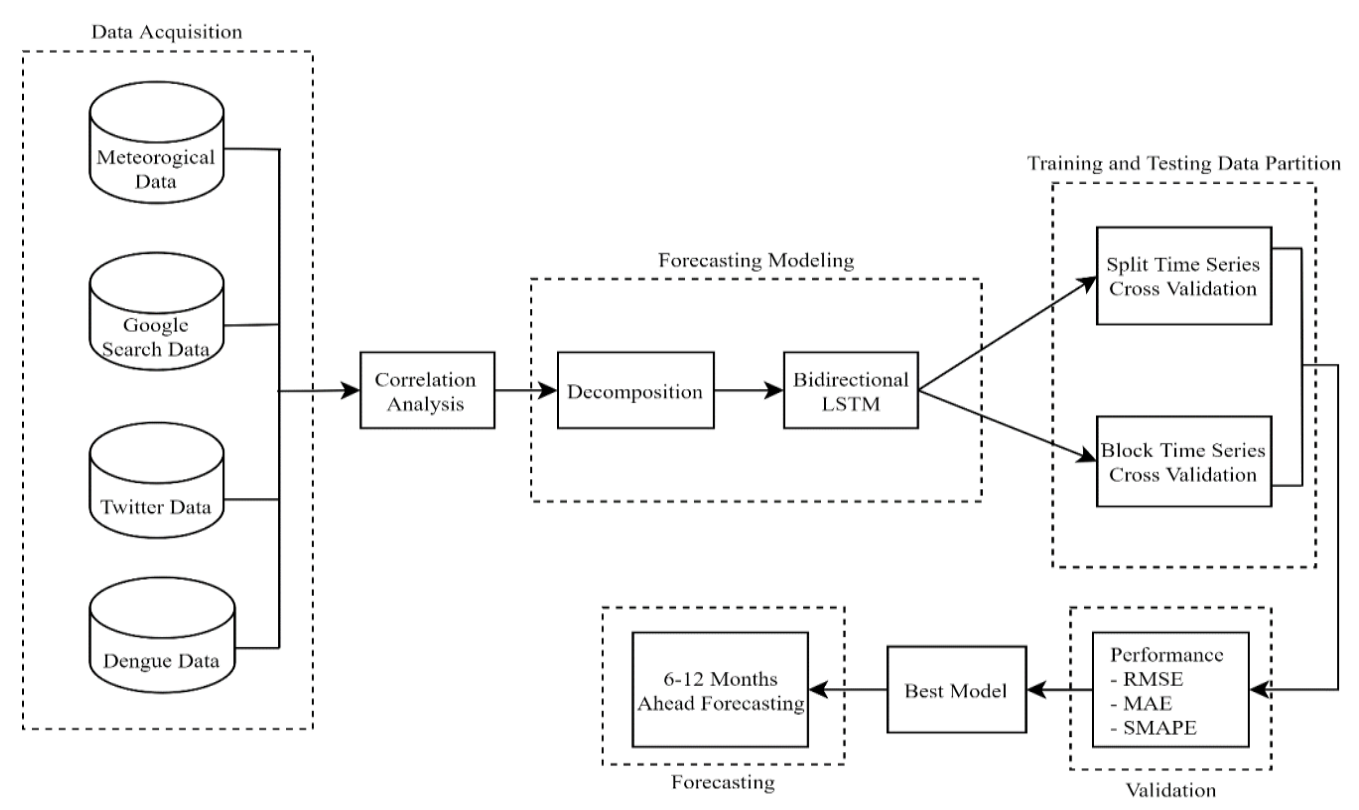

Figure. 2 Summarized framework for the construction of the D-BiLSTM forecasting model for DF cases number

$$
\begin{aligned}
& Y_{t}=f(T, S, R) \\
& Y_{t}=T_{t}+S_{t}+R_{t} \\
& D_{t}=Y_{t}-T_{t} \\
& R_{t}=Y_{t}-T_{t}-S_{t}
\end{aligned}
$$

\subsubsection{BiLSTM modeling}

The LSTM model is a Recurrent Neural Network model that can be used to handle forecasting timeseries data. This LSTM covers the drawbacks of gradient problems from the input applied to the hidden layer, increasing or decreasing significantly during a circular connection. Bidirectional LSTM (BiLSTM) improves LSTM performance by studying both forward and backward input sequences, combining and embedding both meanings in the hidden states. The bidirectional LSTM calculates the hidden forward sequence $\overrightarrow{h_{t}}$ then $\overleftarrow{h_{t}}$ retains information from the future in the backward run, potentially adding a necessary background to the prediction process.

In this study, we use a bidirectional LSTM model with architecture, as displayed in Fig. 3. This architecture consists of two BiLSTM blocks, each with one forward layer (FL) and one backward layer (BL). The $\overrightarrow{s_{l, l}}$ and $\overleftrightarrow{s_{l, l}}$ represents forward hidden state and backward hidden state vectors at layer $1 \in\{1,2\}$ and frequency index $i \in\{1,2, \ldots, 21\}$. These output hidden states are concatenated $\left(\left[\overleftrightarrow{s_{l, l}} ; \overleftrightarrow{S_{l, l}}\right]\right)$ at each frequency index $\mathrm{i}$ and layer 1 before further processing. Also, wi's $(i \in\{1,2, \ldots, 21\})$ are the scalar weights. At each frequency index, we built a two-layer BiLSTM architecture with a residual relation between the outputs of the first and second layers.

In BiLSTM block 1, the input is the result of the decomposition process of each variable denoted by $x_{i}$ with $i=1,2, . ., 21$. The forward layer $\left(\mathrm{FL}_{1}\right)$ provides hidden state $\overleftarrow{S_{1, l}}$. Each hidden state can be denoted as $x$ and $y$, where $\overrightarrow{\varphi_{1}}$ and $\overleftarrow{\varphi_{1}}$ shown parameters of $\mathrm{FL}_{1}$ and $\mathrm{BL}_{1}$.

$$
\begin{aligned}
& \overrightarrow{s_{1, l}}=f\left(\overleftrightarrow{s_{1, l-1}}, x_{i}, \overrightarrow{\varphi_{1}}\right) \\
& \overleftrightarrow{s_{1, l}}=f\left(\overleftrightarrow{s_{1, l+1}}, y_{i}, \overleftrightarrow{\varphi_{1}}\right)
\end{aligned}
$$

The forward and backward hidden states in LSTM cells are now concatenated at each frequency index $(i)$, and the resulting vector is denoted as $\mathrm{s}_{\mathrm{i}, \mathrm{k}}$, where $s_{1, k}=\left[\overleftrightarrow{s_{1, k}} ; \overleftrightarrow{s_{1, k}}\right]$. Meanwhile, in the BiLSTM block 2 , we provide $\mathrm{s}_{1, \mathrm{I}}(\mathrm{i}=1,2, \ldots, 21)$ as input, corresponding forward as $\left(\overrightarrow{s_{2, l}}\right)$, and backward $\left(\overleftarrow{S_{1, k}}\right)$ hidden state vector, which is shown in Eq. (8) and Eq. (9).

$$
\begin{aligned}
& \overrightarrow{s_{2, l}}=f\left(\overleftrightarrow{s_{2, l-1}}, s_{1, i}, \overrightarrow{\varphi_{2}}\right) \\
& \overleftrightarrow{s_{2, l}}=f\left(\overleftarrow{s_{2, l+1}}, s_{1, i}, \overleftrightarrow{\varphi_{2}}\right)
\end{aligned}
$$

The parameters for the $\mathrm{FL}_{2}$ and $\mathrm{BL}_{2}$ are $\overrightarrow{\varphi_{2}}$ and $\overleftarrow{\varphi_{2}}$, respectively. Furthermore, the concatenated output hidden states of BiLSTM $_{2}$ are $s_{2, i}^{\prime}=$ $\left[\overrightarrow{s_{2, l}^{\prime}}, \overleftrightarrow{s_{2, l}^{\prime}}\right]$, where $s_{2, i}^{\prime}=s_{1, i} \oplus s_{2, i}$. We now obtained 


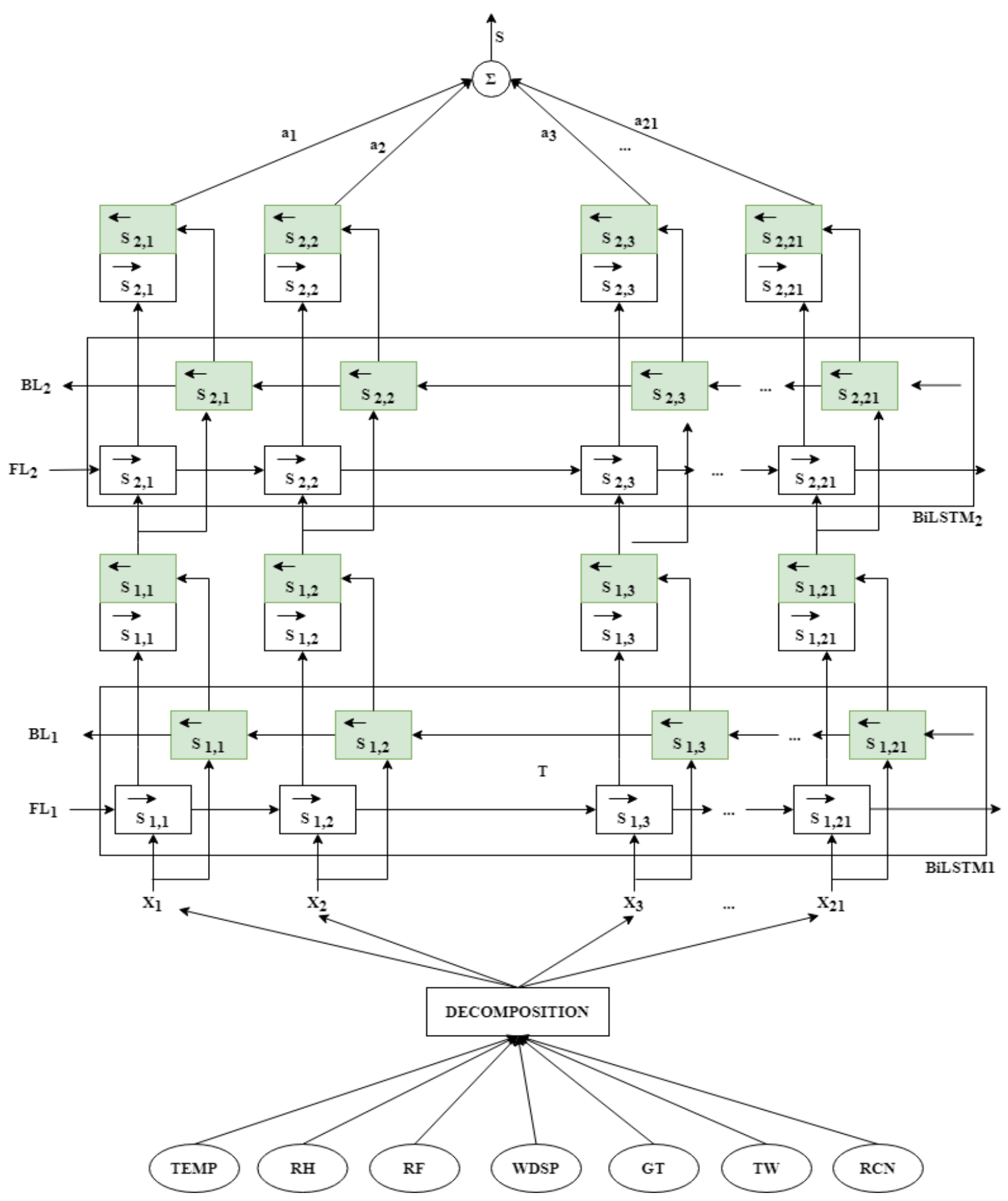

Figure. 3 Decomposition-BiLSTM architecture

single vector $(s)$ by a weighted averaging mechanism as defined in Eq. (10).

$s=\sum_{i=1}^{21} a_{i} s_{i}$

where $a_{i}=\frac{\exp \left(s_{i}^{T} w+b_{w}\right)}{\sum_{i=1}^{21} \exp \left(s_{i}^{T} w+b_{w}\right)} \in(0,1), \mathrm{w}_{\mathrm{i}}, \mathrm{b}_{\mathrm{i}}$ is the trained parameter with other LSTM parameters.

Finally, we get the expected output value $y$, shown in Eq. (11). In Eq. (11), $v$ and $b_{v}$ is another set of trainable weight parameters, and $\sigma$ is the sigmoid function.

$$
y=\sigma\left(v^{T} s+b_{v}\right)
$$




\subsubsection{Cross-validation for model robustness testing}

Cross-validation techniques are used here to maintain the robustness of the D-BiLSTM model against various types and patterns of data in producing forecasts. In addition, this cross-validation technique permits the model to continue to improve its predicting capacity by capturing changes in the relationship between internet browsing data behavior, climate, and the number of reported cases. Two types of cross-validation are used here: five-fold split timeseries cross-validation (STSCV) and blocked timeseries cross-validation (BTSCV). The selection of cross-validation is based on the different patterns of series, types of forecasts, and evaluation objectives.

STSCV divides the training set into two parts at each iteration because the training set is always earlier than the test set. Unlike ordinary cross-validation, which allows the training set to be located after the test set. Meanwhile, BTSCV divides the data by increasing the margin in two places. The first is between the training and testing folds to prevent the model from observing the lag values used as regressors and responses. The second is between the parts used in each iteration to avoid the model from remembering the pattern from iteration to the next iteration [41].

\section{Result and discussion}

This study has two main objectives. The first aim is to find the best combination of D-BiLSTM models to forecast the number of reported cases involving the Google Trends, Twitter, climate, and the number of cases reported variables in the previous period.

At the same time, the second objective is to find out how Google Trends and Twitter data involves in the DF Case forecasting results. There are eight models in each area. Each of these models was tested on different cross-validation scenarios to get a more robust model. The performance of each model in each area is compared with others models [16,34,37]. Then, a forecast for the next 6 and 12 periods is made using the best model for each area so that the Health Office and other stakeholders can plan preventive actions based on the results.

\subsection{Time-series correlation analysis}

The relationship between climate, Google Trends, and Twitter with the DF cases number are shown in Table 1. The correlation coefficient $(r)$ between variables in Table 1 showed correlations between dengue case numbers and other variables. Temperature and rainfall are climate variables that have a small trend correlation among other climate variables. However, even though they are small, they
Tabel 1. The Pearson correlation between the variable of DF cases number with other variables in each area. TEMP: temperature, RH: relative humidity, RF: rainfall, WDSP: wind speed, TW: Twitter; GT: Google Trends, RCN: reported case number

\begin{tabular}{|l|c|c|}
\hline Variable & $\begin{array}{c}\text { Reported } \\
\text { Cases in } \\
\text { Surabaya }\end{array}$ & $\begin{array}{c}\text { Reported } \\
\text { Cases in } \\
\text { Malang }\end{array}$ \\
\hline TEMP & -0.077 & 0.059 \\
\hline RH & 0.293 & 0.373 \\
\hline RF & 0.076 & 0.137 \\
\hline WDSP & 0.128 & -0.21 \\
\hline GT & 0.275 & 0.253 \\
\hline TW & 0.035 & 0.135 \\
\hline RCN Lag 1 & 0.868 & 0.545
\end{tabular}

can affect the number of DHF cases indirectly. It is relevant to previous studies presented by [23, 27, 34]. Temperature affects the growth of mosquitoes as vectors of DF disease [34]. The little correlation value here can be impacted by the fluctuating cases number in certain months where the value can drop significantly. This condition is consistent with [23, 27] that the continuity of relations should be analyzed annually, likewise with rainfall. The maximum rainfall of only $29 \mathrm{~mm}$ in the Malang Regency is too low, so that it is not sufficient to inhibit mosquito growth. On the other hand, rainfall can significantly inhibit mosquito reproduction to a minimum limit of $52 \mathrm{~mm}$ [34]. This condition happened in the Surabaya area, where it once reached a value of $52 \mathrm{~mm}$ so that the correlation value appears to be greater than the Malang district area.

The positive correlation value between Google Trends and reported cases shows that the greater the number of searches on google, the greater the number of reported cases. This positive relationship is relevant to the research conducted by $[6-8,15,17]$. However, in relation to correlation coefficients, this finding is somewhat in contrast to previous studies. Studies conducted by [6,7] show relatively high correlation values $(>0.7)$ between google trends and DF reported at the national level. It could be due to different search query keywords. The level of internet penetration and the keywords used can influence the search frequency [45]. So it is natural that the search correlation value at the level differentiated by the level of penetration tends to be different from the local level without distinction (national level). The level of internet penetration between regions is very different, and local keywords can also be different from others [15]. The decreased correlation results when keywords were separated were also shown by $[8,17]$. However, even though the value is only 0.275 

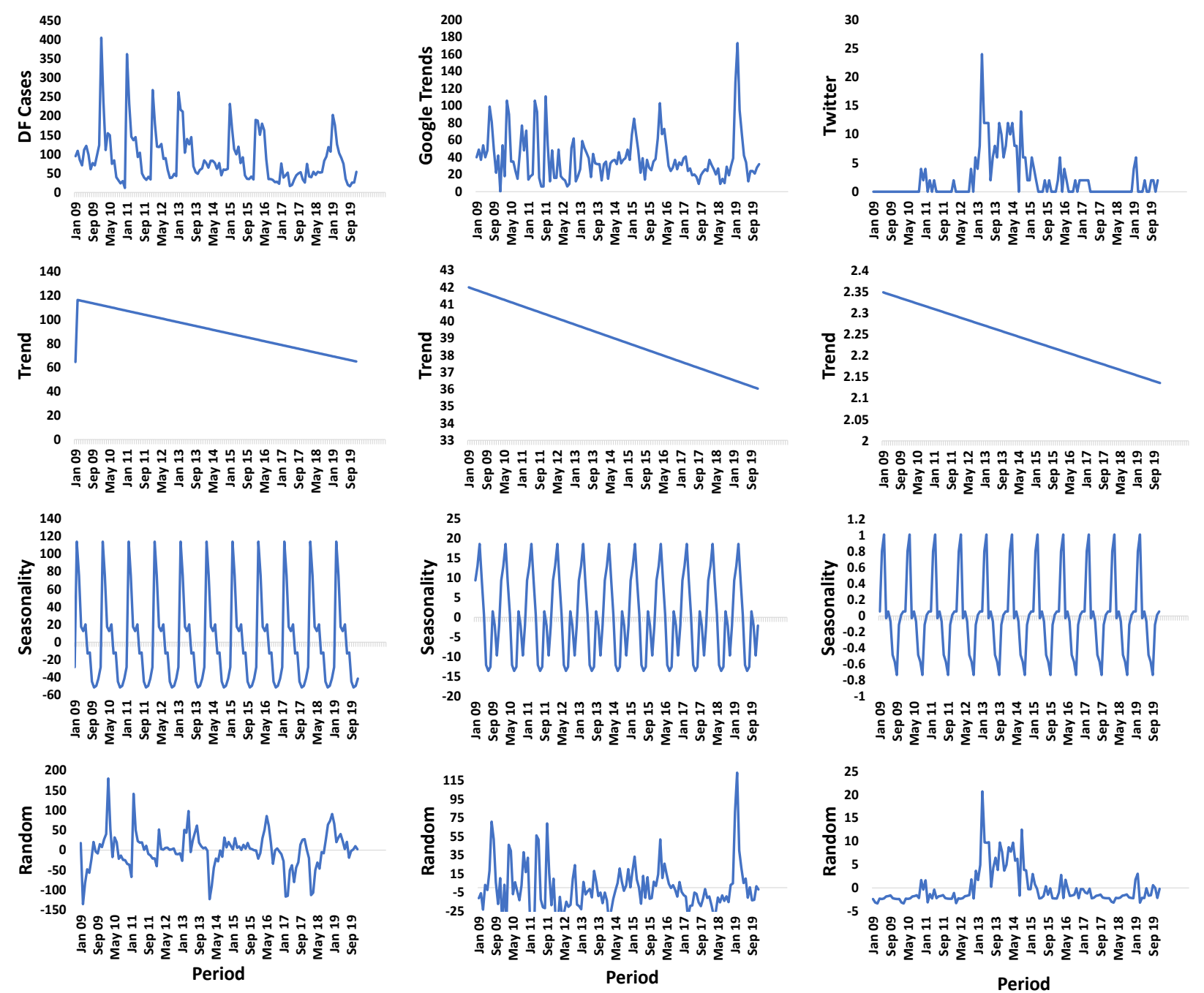

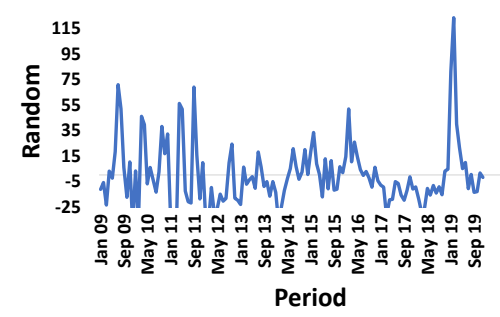

(b)

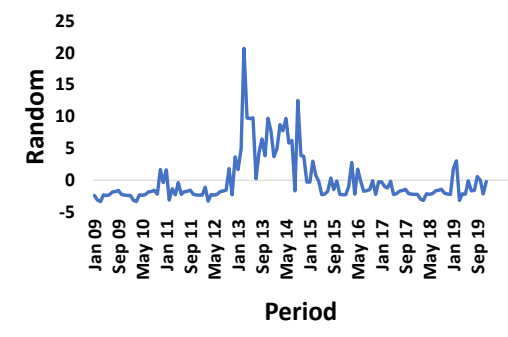

(c)

Figure. 4 Time series decomposition of the Malang area data broken down into trends, seasonality, and random components: (a) reported DF cases, (b) Google Trends, and (c) and Twitter

Table 2. Variable combination scenario implemented in each area. Output: RCN

\begin{tabular}{|c|c|c|}
\hline Scenario & $\begin{array}{c}\text { Best } \\
\text { model }\end{array}$ & Input \\
\hline 1 & M1 & TEMP, RH, RF, WDSP \\
\hline 2 & M2 & TEMP, RH, RF, WDSP, GT \\
\hline 3 & M3 & TEMP, RH, RF, WDSP, TW \\
\hline 4 & M4 & TEMP, RH, RF, WDSP, GT, TW \\
\hline 5 & M5 & TEMP, RH, RF, WDSP, RCN Lag 1 \\
\hline 6 & M6 & TEMP, RH, RF, WDSP, GT, RCN Lag 1 \\
\hline 7 & M7 & TEMP, RH, RF, WDSP, TW, RCN Lag 1 \\
\hline 8 & M8 & TEMP, RH, RF, WDSP, GT, TW, RCN Lag 1 \\
\hline
\end{tabular}

for the Surabaya area and 0.253 in Malang Regency, this value is still said to affect the 0.01 level significantly.

Although Twitter's influence is not as strong as Google Trends, the positive correlation still shows that an increase also followed the rise in the number of Twitter in the number of DF cases reported. Twitter in Indonesia in the early study period was still rare and had only increased in the last three years [32]. 
Table 3. The result of D-BiLSTM models. Model 1-4 excludes the DF cases number at lag 1 in the independent variable combination. Model 5-8 includes the DF cases number at lag 1 in the independent variable combination.

\begin{tabular}{|c|c|c|c|c|c|c|c|c|c|c|c|c|}
\hline \multirow{2}{*}{ Model } & \multicolumn{3}{|c|}{ Surabaya-STSCV } & \multicolumn{3}{c|}{ Malang-STSCV } & \multicolumn{3}{c|}{ Surabaya-BTSCV } & \multicolumn{3}{c|}{ Malang-BTSCV } \\
\cline { 2 - 14 } & RMSE & MAE & SMAPE & RMSE & MAE & SMAPE & RMSE & MAE & SMAPE & RMSE & MAE & SMAPE \\
\hline M1 & 100.304 & 72.683 & 49.174 & 86.422 & 65.887 & 53.056 & 109.387 & 73.294 & 48.427 & 89.201 & 68.270 & 49.053 \\
\hline M2 & 100.238 & 72.636 & 49.204 & 83.986 & 62.827 & 48.097 & 109.623 & 73.530 & 48.745 & 88.965 & 68.001 & 48.730 \\
\hline M3 & 100.288 & 72.710 & 49.208 & 83.870 & 62.719 & 47.949 & 109.485 & 73.381 & 48.774 & 89.515 & 68.633 & 49.584 \\
\hline M4 & 100.034 & 72.463 & 48.751 & 84.006 & 62.907 & 48.218 & 109.583 & 73.500 & 48.838 & 89.085 & 68.153 & 48.964 \\
\hline M5 & 100.109 & 72.525 & 49.131 & 84.187 & 63.084 & 48.597 & 109.824 & 73.782 & 49.419 & 89.579 & 68.674 & 49.741 \\
\hline M6 & 100.060 & 72.560 & 48.782 & 83.705 & 62.508 & 47.781 & 109.887 & 73.854 & 49.491 & 89.394 & 68.459 & 49.430 \\
\hline M7 & 100.142 & 72.513 & 49.197 & 83.712 & 62.546 & 47.705 & 109.728 & 73.652 & 49.003 & 89.316 & 68.383 & 49.026 \\
\hline M8 & 100.184 & 72.685 & 49.101 & 83.173 & 61.955 & 46.816 & 109.975 & 73.966 & 49.635 & 89.508 & 68.608 & 49.586 \\
\hline
\end{tabular}

Table 4. The result of a 12-months forecast using D-BiLSTM models. Model 1-4 excludes the reported DF cases at lag 1. Model 5-8 includes the DF cases number at lag 1. Mark-bold models denote the best model

\begin{tabular}{|c|c|c|c|c|c|c|c|c|c|}
\hline Area & Error & M1 & M2 & M3 & M4 & M5 & M6 & M7 & M8 \\
\hline \multirow{4}{*}{ Surabaya } & RMSE & 53.785 & 44.491 & 68.861 & 68.712 & $\mathbf{3 0 . 1 1 1}$ & 32.694 & 68.883 & 68.763 \\
\cline { 2 - 10 } & MAE & 51.541 & 42.390 & 65.887 & 65.754 & 19.110 & 25.986 & 65.907 & 65.798 \\
\cline { 2 - 11 } & SMAPE & 0.548 & 0.502 & 0.592 & 0.592 & 0.310 & 0.389 & 0.592 & 0.592 \\
\hline \multirow{4}{*}{ Malang } & RMSE & 32.723 & 30.749 & 31.509 & 33.001 & 33.885 & 29.892 & $\mathbf{2 8 . 6 5 1}$ & 30.157 \\
\cline { 2 - 10 } & MAE & 29.117 & 27.253 & 27.901 & 29.377 & 30.125 & 26.467 & 25.727 & 26.700 \\
\cline { 2 - 10 } & SMAPE & 0.202 & 0.191 & 0.195 & 0.203 & 0.207 & 0.187 & 0.183 & 0.189 \\
\hline
\end{tabular}

Table 5. The t-value result in the hypothesis test by comparing models that exclude and include the internet query variables. The threshold used is 1.645 with a significant level of 0.05 . One-tailed test, $\mathrm{H}_{0}: M_{i}-M_{j} \geq 0, i=1,5 ; j=$

$$
2,3,4,6,7,8
$$

\begin{tabular}{|c|c|c|c|c|}
\hline \multirow{2}{*}{ Paired Models } & \multicolumn{2}{|c|}{ Surabaya } & \multicolumn{2}{c|}{ Malang } \\
\cline { 2 - 5 } & t-value & Conclusion & t-value & Conclusion \\
\hline M1 - M2 & 1.885 & M2 is better than M1 & 6.049 & M2 is better than M1 \\
\hline M1 - M3 & -6.831 & M3 is not better than M1 & 11.407 & M3 is better than M1 \\
\hline M1 - M4 & -6.729 & M4 is not better than M1 & -19.932 & M4 is not better than M1 \\
\hline M5 - M6 & -50.510 & M6 is not better than M5 & 18.622 & M6 is better than M5 \\
\hline M5 - M7 & -10.674 & M7 is not better than M5 & 19.989 & M7 is better than M5 \\
\hline M5 - M8 & -50.787 & M8 is not better than M5 & 17.874 & M8 is better than M5 \\
\hline
\end{tabular}

\subsection{Decomposition-bidirectional LSTM (D- BiLSTM) model}

The decomposition of each independent variable produces trend, seasonality, and random components. The results of the vector decomposition of the number of registered DF events, Google Trends, and Twitter are displayed in Fig. 4. The trend component indicates that the number of reported DF cases has a declining trend and Twitter and Google Trends variables. The many jagged random components, especially in the DF case number, indicate that there are events that cannot be explained by the periodicity of this data, which other factors can cause. Then, the seasonal component seems to repeat itself, and this occurs every close to 12 months.
This finding is relevant to previous research conducted in Indonesia [6]. This period is identical to the time of the rainy season. This seasonal period also looks similar to Google Trends and Twitter. Note that the seasonal and random components have negative values due to the decomposition and relative to the moving average month length. These negative scores on the random and seasonal components are for comparison and do not imply negative case numbers or search numbers.

DF cases data from Malang and Surabaya, climate data, local search queries, and social media are used to construct the BiLSTM model. Table 2 presents eight scenarios of variable combinations that produce the eight best models in each scenario. These scenarios are carried out in each area. Models from 
Scenario 1-4 exclude dengue data reported case number at lag 1 [30], while models from Scenario 58 include reported DF case number at lag 1 [7].

In each scenario, tests were carried out using two types of 5-fold cross-validation, namely STSCV and BTSCV. This cross-validation is carried out in DBiLSTM using 500 epochs, the mean square error loss function, and the sigmoid activation function. The other parameters were: 4 hidden layers, 32 units per hidden layer, 32 batch size, 0.5 drop out, 0.005 learning rate. Meanwhile, the optimizer used is the best optimizer selected from the optimization algorithm that produces the smallest root mean square error. The optimization algorithms were Adam, Adadelta, Stochastic Gradient Descent (SGD), Adagrad, Adamax, Nadam, RMSprop, and Ftrl. Testing was carried out using Python 3.5.3 includes the Keras module.

The best performing model in each area using two types of cross-validation with the best optimizer isshown in Table 3. The performance metrics used are root mean square error (RMSE), mean absolute error (MAE), and root mean absolute percentage. error (SMAPE) [13].

The performance of each model in Table 3 is the best average of each fold on STSCV and BTSCV. This condition is because they have experimented with the testing data in each area. The metric used is RMSE, and the best model has the smallest RMSE [40]. The use of two kinds of cross-validation aims to make the model learn with more data composition. In this study, the average performance of the DBiLSTM model using STSCV has better performance than BTSCV. This performance is proper for both models. This condition can be caused because the conditions at the time of STSCV could get a more significant proportion of training data than BTSCV. So, the model can learn better with more data. It is relevant to previous studies, stating that training using more data will increase the model's performance.

Next, the retrain model uses cross-validation to get the results of forecasting data validation. The results of the retrain process are predicting the number of DF cases for data validation in the next period again. The results of the best model performance in each area are shown in Table 4.

Table 4 presents that the performance of the DBiLSTM model involving DF case lag 1 produces better performance than the model without involving these variables. These findings are consistent with those presented in the experimental data testing shown in Table 3. This condition can occur because the DF case lag 1 has a very high correlation with the current DF case. Highly correlated variables can improve the performance of a model [6]. Table 4 also shows that adding Google Trends and Twitter variables can increase or decrease forecasting performance. In the Surabaya area, which represents high internet penetration rates, models without Google Trends and Twitter data have the best performance. Whereas in the Malang Regency area, a model that involves all data simultaneously is the best model.

The RMSE value in Table 4 also shows a different effect in areas with varying levels of internet penetration. In areas with high penetration, namely Surabaya, data on google trends and twitter is not proven to reduce error significantly. The RMSE value is getting bigger by involving these two variables, except M2, which requires Google Trends without DF cases in the previous period. M2 decreased by 9,295 . For Malang Regency as the representative of areas with low internet penetration, RMSE in M2 and M3 looks lower than M1. It does not apply to M4. However, M6, M7, and M8 have a smaller RMSE value than M5. M2 has a decrease in the average RMSE of 1,099. A comparison test of two samples was carried out to determine whether the RMSE difference is significant enough to increase or decrease performance. Table 5 shows the $t$-student values obtained by paired samples test carried out on sample pairs M1 with M2, M3, M4, and M5 with M6, M7, M8.

The t-value in Table 5 shows that the addition of Twitter as a predictor variable is not proven to improve forecasting performance. However, for Google Trends, it is still quite significant to improve performance. However, if Google Trends include together with Twitter, it is still not enough to increase performance. It is somewhat different from the Malang Regency area, where the internet penetration rate is lower. The addition of the variables Google Trends and Twitter proved to significantly increase performance except when the two data were involved together and without involving the DF case in the previous period.

These results cannot be compared with previous studies because, to the best of the authors' knowledge of the DF case, most previous studies did not pay attention to the differences between areas with different internet penetration rates and local searches. However, if the previous research used national-level data, the results of this study are partly relevant. Previous research conducted by $[8,17]$ stated that using data at the national aggregate level, Google Trends data can improve forecasting quality. This finding is relevant for models in low internet penetration but not appropriate for models in high penetration areas with DF case lag 1. Several 
Table 6. Comparison of model performance in areas with higher internet penetration rates in Surabaya. The markbold numbers represent the two highest-performing models.

\begin{tabular}{|l|c|c|c|c|c|c|c|c|c|}
\hline Method & Error & $\mathbf{M 1}$ & $\mathbf{M 2}$ & $\mathbf{M 3}$ & $\mathbf{M 4}$ & $\mathbf{M 5}$ & $\mathbf{M 6}$ & M7 & M8 \\
\hline \multirow{2}{*}{$\begin{array}{l}\text { LASSO } \\
{[34]}\end{array}$} & RMSE & 139.596 & $\mathbf{1 4 1 . 8 8 2}$ & $\mathbf{1 4 0 . 1 8 5}$ & $\mathbf{1 3 8 . 1 8 9}$ & $\mathbf{7 4 . 5 1 9}$ & $\mathbf{7 5 . 1 5 9}$ & $\mathbf{7 5 . 8 9}$ & $\mathbf{7 7 . 7 6 4}$ \\
\cline { 2 - 10 } & MAE & 92.529 & 91.665 & 92.256 & 90.235 & 44.263 & 46.433 & 46.604 & 46.730 \\
\cline { 2 - 10 } & SMAPE & 0.370 & 0.384 & 0.355 & 0.381 & 0.332 & 0.335 & 0.318 & 0.334 \\
\hline \multirow{2}{*}{$\begin{array}{l}\text { ARIMAX } \\
{[18]}\end{array}$} & RMSE & $\mathbf{1 1 4 . 9 0 3}$ & 168.958 & 228.106 & 216.911 & 128.291 & 125.818 & 141.761 & 128.291 \\
\cline { 2 - 10 } & MAE & 111.948 & 159.291 & 222.477 & 210.725 & 86.542 & 82.423 & 99.836 & 86.542 \\
\cline { 2 - 10 } & SMAPE & 0.621 & 0.704 & 0.747 & 0.740 & 0.323 & 0.313 & 0.341 & 0.323 \\
\hline \multirow{3}{*}{ NN $[36]$} & RMSE & 177.188 & 175.520 & 176.496 & 174.682 & 172.270 & 170.071 & 171.489 & 171.795 \\
\cline { 2 - 10 } & MAE & 100.390 & 98.878 & 99.413 & 97.735 & 99.054 & 96.885 & 97.672 & 97.555 \\
\cline { 2 - 10 } & SMAPE & 0.173 & 0.181 & 0.177 & 0.185 & 0.168 & 0.181 & 0.179 & 0.177 \\
\hline \multirow{3}{*}{$\begin{array}{l}\text { SVM } \\
{[36]}\end{array}$} & RMSE & 155.971 & 156.673 & 156.281 & 156.553 & 156.264 & 156.334 & 156.383 & 156.846 \\
\cline { 2 - 10 } & MAE & 81.837 & 82.336 & 81.628 & 82.207 & 81.635 & 81.918 & 82.234 & 82.260 \\
\cline { 2 - 10 } D- & SMAPE & 0.331 & 0.331 & 0.332 & 0.328 & 0.333 & 0.332 & 0.330 & 0.330 \\
\hline BiLSTM & RMSE & $\mathbf{5 3 . 7 8 5}$ & $\mathbf{4 4 . 4 9 1}$ & $\mathbf{6 8 . 8 6 1}$ & $\mathbf{6 8 . 7 1 2}$ & $\mathbf{3 0 . 1 1 1}$ & $\mathbf{3 2 . 6 9 4}$ & $\mathbf{6 8 . 8 8 3}$ & $\mathbf{6 8 . 7 6 3}$ \\
\cline { 2 - 9 } & MAE & 51.541 & 42.390 & 65.887 & 65.754 & 19.110 & 25.986 & 65.907 & 65.798 \\
\cline { 2 - 9 } & SMAPE & 0.548 & 0.502 & 0.592 & 0.592 & 0.310 & 0.389 & 0.592 & 0.592 \\
\hline
\end{tabular}

Table 7. Comparison of model performance in areas with lower internet penetration rates in Malang. The mark-bold numbers represent the two highest-performing models.

\begin{tabular}{|l|c|c|c|c|c|c|c|c|c|}
\hline Method & Error & M1 & M2 & M3 & M4 & M5 & M6 & M7 & M8 \\
\hline \multirow{2}{*}{$\begin{array}{l}\text { LASSO } \\
{[34]}\end{array}$} & RMSE & $\mathbf{6 5 . 2 7 3}$ & $\mathbf{6 3 . 7 1 8}$ & $\mathbf{6 6 . 8 5 1}$ & $\mathbf{6 3 . 5 0 2}$ & $\mathbf{5 6 . 7 8 2}$ & $\mathbf{5 7 . 7 5 3}$ & $\mathbf{5 7 . 1 0 2}$ & $\mathbf{5 8 . 2 6 7}$ \\
\cline { 2 - 10 } & MAE & 47.053 & 45.088 & 48.153 & 44.488 & 32.646 & 35.058 & 33.353 & 34.653 \\
\cline { 2 - 10 } & SMAPE & 0.320 & 0.314 & 0.311 & 30.675 & 33.924 & 32.057 & 32.872 & 32.405 \\
\hline \multirow{3}{*}{$\begin{array}{l}\text { ARIMAX } \\
{[18]}\end{array}$} & RMSE & 330.946 & 126.736 & 183.013 & 172.123 & 237.541 & 122.822 & 207.708 & 165.942 \\
\cline { 2 - 10 } & MAE & 78.057 & 112.082 & 174.602 & 162.850 & 112.599 & 65.229 & 98.943 & 82.594 \\
\cline { 2 - 10 } & SMAPE & 41.997 & 36.165 & 39.720 & 38.580 & 29.403 & 24.776 & 28.209 & 25.441 \\
\hline \multirow{4}{*}{ NN [36] } & RMSE & 104.763 & 102.854 & 103.429 & 101.395 & 102.262 & 100.385 & 100.805 & 101.760 \\
\cline { 2 - 10 } & MAE & 80.740 & 79.316 & 79.595 & 77.647 & 79.339 & 77.733 & 77.783 & 78.662 \\
\cline { 2 - 9 } & SMAPE & 0.138 & 0.139 & 0.144 & 15.751 & 13.643 & 14.905 & 15.168 & 14.571 \\
\hline \multirow{3}{*}[36]{} & RMSE & 90.376 & 88.456 & 87.828 & 88.715 & 69.874 & 68.790 & 68.927 & 68.979 \\
\cline { 2 - 9 } & MAE & 50.535 & 50.141 & 49.669 & 51.043 & 48.172 & 47.121 & 47.334 & 47.232 \\
\cline { 2 - 9 } & SMAPE & 0.301 & 0.303 & 0.301 & 31.152 & 29.280 & 29.357 & 29.237 & 29.301 \\
\hline \multirow{2}{*}{$\begin{array}{l}\text { B- } \\
\text { BiLSTM }\end{array}$} & RMSE & $\mathbf{3 2 . 7 2 3}$ & $\mathbf{3 0 . 7 4 9}$ & $\mathbf{3 1 . 5 0 9}$ & $\mathbf{3 3 . 0 0 1}$ & $\mathbf{3 3 . 8 8 5}$ & $\mathbf{2 9 . 8 9 2}$ & $\mathbf{2 8 . 6 5 1}$ & $\mathbf{3 0 . 1 5 7}$ \\
\cline { 2 - 9 } & MAE & 29.117 & 27.253 & 27.901 & 29.377 & 30.125 & 26.467 & 25.727 & 26.700 \\
\cline { 2 - 9 } & SMAPE & 0.202 & 0.191 & 0.195 & 0.203 & 0.207 & 0.187 & 0.183 & 0.189 \\
\hline
\end{tabular}

explanations can be given regarding this contradiction. First, a prior research used a forecasting model that was analyzed per keyword [17], whereas, in this study, we used an aggregate of several keywords with the highest number of uses. This selection certainly causes different types of keywords to be used. Furthermore, varying types of keywords can cause accuracy differences [15]. In addition, [8] does not involve climate variables.

The only variables involved are reported data and Google Trends. It is relevant to what was stated by [17] that the differences in the variables involved could affect the model's performance. In addition, the random values of Google Trends and the very random Twitter, as shown in Fig. 2, can also influence this finding. Although the correlation is proven to be significant, it cannot improve the prediction result [21], likewise with Twitter data. However, let's look at the cases of other diseases. The findings of this study for the Surabaya area are relevant to those expressed by [21], who stated that online search data does not significantly improve forecasting performance.

To see how the performance position of the DBILSTM model is compared to others, the DBiLSTM model is compared with other models that have been used in previous studies. The results of the proposed performance model and its comparison in areas with a higher internet penetration rate - 
Surabaya- are shown in Table 6. In contrast, areas with lower internet penetration are shown in Table 7. Table 6 and Table 7 show that the D-BiLSTM model has better performance than its rival model. The Lasso model is the second-best model, where even though the average RMSE is still above 100, it is still lower than the others. The outcomes of this research indicate that the D-BiLSTM model succeeded in reducing the average error. D-BiLSTM reduces RMSE by 93,129 and 94,054 for M1 in Surabaya and Malang. Thereafter 93,129 and 50,950, for M2, 106,406 and 41,419 for M3, 102,872 and 37,721 for M4, 102,725 and 86,504 for M5. Subsequently, 99,152 and 54,743 for the M6, 67,498 and 39,753 for the M7, 64,911 and 29,974 for the M8. The M5 model is the best model for D-BiLSTM in the Surabaya region, having a SMAPE of 0.310 and an MAE of 19,110. In the Malang area, the best model, M7, has a SMAPE value of 0.183 and MAE 25,727. These values are still below the $10 \%$ range of the Surabaya and Malang data intervals so that the forecasting results can still be said to be excellent.

The reported DF cases and forecast with the DBiLSTM model and Lasso comparison with the best performance are shown in Fig. 5. Forecasting involves variables following the best models of DBiLSTM, namely M5 in Surabaya and M7 in Malang. Fig. 5 shows that the forecast results of the DBiLSTM model are closer to the actual data than the LASSO model. The trend and seasonal factors can influence this phenomenon, and random factors of dengue fever are associated with climate variables, Google Trends, and Twitter. The D-BiLSTM model studies data based on these three components, as shown in Fig. 2, so that the results can be closer to the actual data than studying one factor as a single value.

If observed in Fig. 2, the trend between reported DF and Twitter in the Malang area is the same.

Similarly, a seasonal pattern where the period is 12 months experienced the peak of events in February 2019. Then for the random component between Twitter rose also in February 2019 where previously tended to low. However, the random pattern for more varied DF cases up and down is drastic and up in December 2018. It is a similarity that makes the model easier to learn. This condition is the opposite in Surabaya. Here, models with Twitter and Google

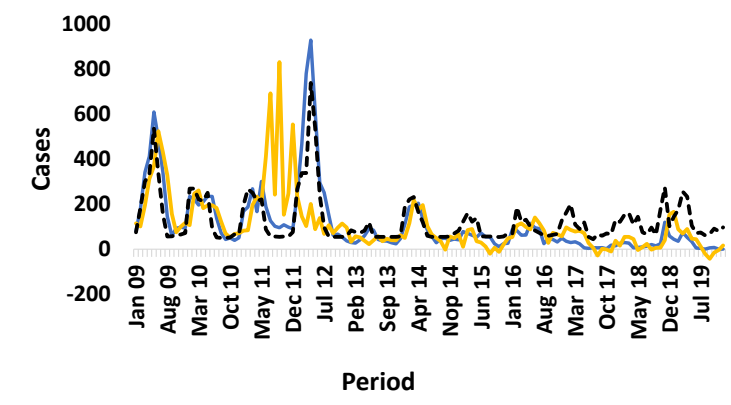

— Reported Cases — Forecast of LASSO ---Forecast of D-BiLSTM

(a)

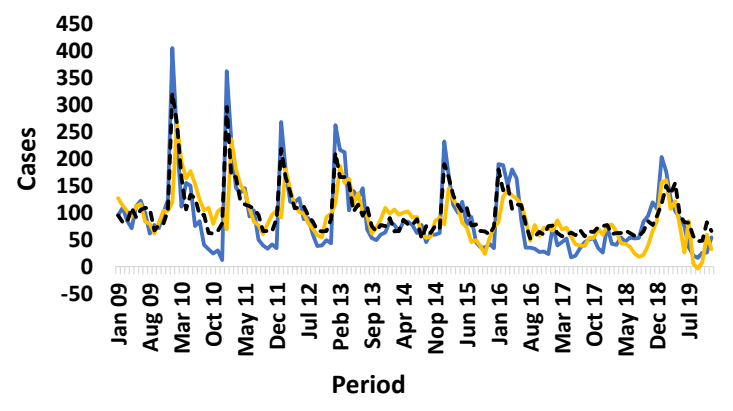

Figure. 5 Comparison of reported DF case data and forecast results with the D-BiLSTM and Lasso models in each area: (a) Surabaya (b) Malang.

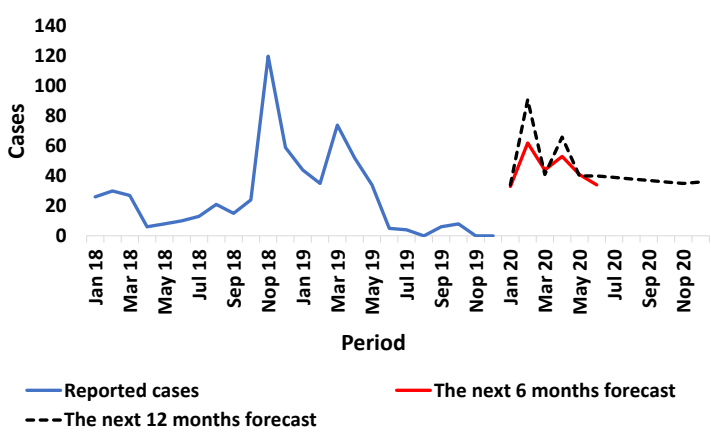

(a)

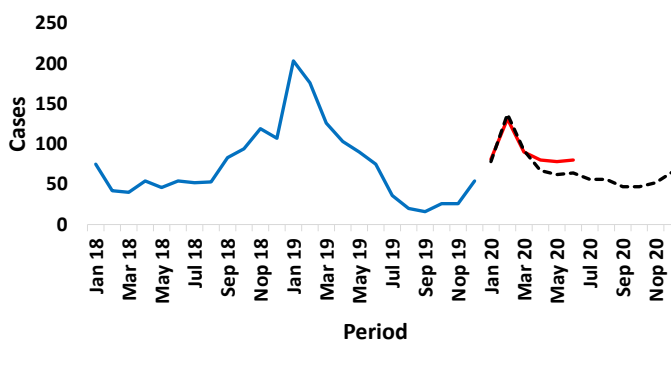

- Reported cases
---The next 12 months forecast

(b)

Figure. 6 Forecast results for the next 6 and 12 months using the D-BiLSTM model in each area: (a) Surabaya (b) Malang. 
Trends are not the best models. If observed, the pattern of random DF cases reported more sloping in recent years, but this contrasts to Twitter and Google Trends that tend to increase. Similarly, the value of reported DF cases tends to decrease but rises for Twitter and Google Trends. This condition makes the D-BiLSTM model more responsive.

\subsection{The next 6-12 periods forecasting}

Action planning to avoid an increase in the number of cases can be done appropriately by forecasting the number of cases for several months ahead. The forward forecast period used is 6 and 12 months. This choice is based on the need for budget planning and actions taken in the study area [43]. Comparison of D-BiLSTM forecast skills for nowcast, six months, and 12 months ahead of the forecast using Model 5 is presented in Fig. 6 (a) and Fig. 6 (b).

Fig. 6 depicts the forecast patterns for the next 6 and 12 months in all areas have a similar pattern. However, relative to the previous 12 months, the forecasting results for the next six months tend to be identical to the value in the same month last year. This situation was relevant to the study presented by [13]. The more extended the forecast period in the future, there is a tendency for more extensive errors, which means that the pattern will be more different than the short term.

\section{Conclusion and future works}

To the best of our experience, this is the first study evaluating forecasting performance using internet query and social media data for dengue fever which separates searches based on differences in internet penetration rates between regions. Previous studies mostly used tracing data collected nationally, which was linked to the number of national cases, so that only policies that could be taken were at the national level. Whereas in reality, regional or local parties also need to prepare policies that are per the conditions of their respective regions where these conditions are not necessarily the same when viewed nationally.

In this research, the influence of Google Trends and Twitter variables is presented in 8 scenarios that produce 1440 models based on the combination of variables, the type of cross-validation, and the optimizer used. There are eight best models in each area. Twitter data is more influential on DF cases reported in areas with higher penetration rates. Google Trends is more significant than Twitter in areas with low and high internet penetration. Even though it is influential, forecasting involving Google
Trends and Twitter at the local level does not necessarily increase forecasting performance, especially for areas with high internet penetration. Dengue fever forecasting is reported to have the best performance when it involves climate variables without Google Trends and Twitter variables in areas with high internet penetration rates. Meanwhile, the best model for low internet penetration rates involves climate, Twitter, and DF in the previous lag.

Based on correlation and forecasting, Google Trends and Twitter are not used to replace the reported data. Still, based on the similarity of the reported dengue fever case, Google Trends, and Twitter decomposition patterns, they may help describe the public's response to disease behavior. However, Twitter and Google Trends can be valuable sources of information. Nevertheless, this study has limitations, namely the limited number of areas.

For this reason, in the following research, the combination model obtained will be applied to different and more local areas. In addition, the analysis is focused on separating the number of low and high cases so that it is more apparent how the contribution of social media data to the development of DF cases.

\section{Conflicts of interest}

The authors declare no conflict of interest.

\section{Author contributions}

Wiwik Anggraeni: conceptualization, methodology, formal analysis, writing - original draft preparation and editing. Eko Mulyanto Yuniarno: conceptualization, validation, formal analysis, writing - review. Reza Fuad Rachmadi: data curation, validation, writing - review. Mauridhi Hery Purnomo: supervision, conceptualization, formal analysis, writing-review. All authors read and approved the final manuscript.

\section{Acknowledgments}

We would like to express our gratitude to the Ministry of Research, Technology, and Higher Education of the Republic of Indonesia for providing research funding through the Doctoral Dissertation Research grant scheme, University Center of Excellence on Artificial Intelligence for Healthcare and Society (UCE AIHeS), and the Malang Regency Public Health Services for their assistance.

\section{References}

[1] W. Int, "Dengue and severe dengue", 2018. https://www.who.int/news-room/fact- 
sheets/detail/dengue-and-severe-dengue (accessed Mar. 27, 2019).

[2] WHO, "WHO | Dengue guidelines for diagnosis, treatment, prevention and control: new edition", WHO, 2017. https://www.who.int/rpc/guidelines/978924154 7871/en/ (accessed Mar. 28, 2019).

[3] "WHO 29 July 2016, vol. 91, 30 (pp. 349-364)", WHO.

http://www.who.int/wer/2016/wer9130/en/ (accessed Nov. 25, 2019).

[4] F. Y. Nejad and K. D. Varathan, "Identification of significant climatic risk factors and machine learning models in dengue outbreak prediction", BMC Medical Informatics and Decision Making, Vol. 21, No. 1, p. 141, 2021.

[5] B. H. D. P2P, "Kesiapsiagaan Menghadapi Peningkatan Kejadian Demam Berdarah Dengue Tahun 2019 [Preparedness for Facing the Increased Incidence of Dengue Hemorrhagic Fever in 2019]| Direktorat Jendral P2P." http://p2p.kemkes.go.id/kesiapsiagaanmenghadapi-peningkatan-kejadian-demamberdarah-dengue-tahun-2019/ (accessed Mar. 27, 2020).

[6] A. Husnayain, A. Fuad, and L. Lazuardi, "Correlation between Google Trends on dengue fever and national surveillance report in Indonesia," Global Health Action, Vol. 12, No. 1, p. 1552652, 2019.

[7] S. Yang, S. C. Kou, F. Lu, J. S. Brownstein, N. Brooke, and M. Santillana, "Advances in using Internet searches to track dengue", PLoS Computational Biology, Vol. 13, No. 7, 2017.

[8] R. A. Strauss, J. S. Castro, R. Reintjes, and J. R. Torres, "Google dengue trends: An indicator of epidemic behavior. The Venezuelan Case", International Journal of Medical Informatics, Vol. 104, pp. 26-30, 2017.

[9] R. Strauss, E. Lorenz, K. Kristensen, D. Eibach, J. Torres, J. May, and J. Castro, "Investigating the utility of Google trends for Zika and Chikungunya surveillance in Venezuela", $B M C$ Public Health, Vol. 20, 2020.

[10] "World Telecommunication/ICT Indicators Database." https://www.itu.int/en/ITUD/Statistics/Pages/publications/wtid.aspx (accessed Feb. 26, 2021).

[11]E. Hagg, V. S. Dahinten, and L. M. Currie, "The emerging use of social media for health-related purposes in low and middle-income countries: A scoping review," International Journal of Medical Informatics, Vol. 115, pp. 92-105, 2018.
[12]E. H. Chan, V. Sahai, C. Conrad, and J. S. Brownstein, "Using Web Search Query Data to Monitor Dengue Epidemics: A New Model for Neglected Tropical Disease Surveillance", PLoS Neglected Tropical Diseases, Vol. 5, No. 5, p. e1206, 2011.

[13]P. Rangarajan, S. K. Mody, and M. Marathe, "Forecasting dengue and influenza incidences using a sparse representation of Google trends, electronic health records, and time series data", PLoS Computational Biology, Vol. 15, No. 11, 2019.

[14]Y. Zhang, H. Bambrick, K. Mengersen, S. Tong, and $\mathrm{W}$. $\mathrm{Hu}$, "Using Google Trends and ambient temperature to predict seasonal influenza outbreaks", Environment International, Vol. 117, pp. 284-291, 2018.

[15]P. Guo, L. Wang, Y. Zhang, G. Luo, Y. Zhang, C. Deng, Q. Zhang, and Q. Zhang, "Can internet search queries be used for dengue fever surveillance in China?", International Journal of Infectious Diseases, Vol. 63, pp. 74-76, 2017.

[16]A. Wilder-Smith, E. Cohn, D. C. Lloyd, Y. Tozan, and J. S. Brownstein, "Internet-based media coverage on dengue in Sri Lanka between 2007 and 2015", Global Health Action, Vol. 9, No. 1, p. 31620, 2016.

[17]W. Anggraeni and L. Aristiani, "Using Google Trend data in forecasting number of dengue fever cases with ARIMAX method case study: Surabaya, Indonesia", In: Proc. of 2016 International Conference on Information Communication Technology and Systems (ICTS), Surabaya, Indonesia, pp. 114-118, 2016.

[18]H. T. Ho, T. M. Carvajal, J. R. Bautista, J. D. R. Capistrano, K. M. Viacrusis, L. F. T. Hernandez, and K. Watanabe, "Using Google Trends to Examine the Spatio-Temporal Incidence and Behavioral Patterns of Dengue Disease: A Case Study in Metropolitan Manila, Philippines", Tropical Medicine and Infectious Disease, Vol. 3, No. 4, 2018.

[19] Y. Teng, D. Bi, G. Xie, Y. Jin, Y. Huang, B. Lin, $X$. An, D. Feng, and Y. Tong, "Dynamic Forecasting of Zika Epidemics Using Google Trends", PLoS ONE, Vol. 12, No. 1, 2017.

[20]M. Verma, K. Kishore, M. Kumar, A. R. Sondh, G. Aggarwal, and S. Kathirvel, "Google Search Trends Predicting Disease Outbreaks: An Analysis from India", Healthcare Informatics Research, Vol. 24, No. 4, p. 300, 2018.

[21]M. Kapitány-Fövény, T. Ferenci, Z. Sulyok, J. Kegele, H. Richter, I. V. Nagy, and M. Sulyok, "Can Google Trends data improve forecasting of 
Lyme disease incidence?", Zoonoses Public Health, Vol. 66, No. 1, pp. 101-107, 2019.

[22]Z. Du, L. Xu, W. Zhang, D. Zhang, S. Yu, and Y. Hao, "Predicting the hand, foot, and mouth disease incidence using search engine query data and climate variables: an ecological study in Guangdong, China”, BMJ Open, Vol. 7, No. 10, 2017.

[23] G. Zhu, T. Liu, J. Xiao, B. Zhang, T. Song, Y. Zhang, L. Lin, Z. Peng, A. Deng, W. Ma, and Y. Hao, "Effects of human mobility, temperature and mosquito control on the spatiotemporal transmission of dengue", Science of the Total Environment, Vol. 651, pp. 969-978, 2019.

[24]A. A. E. Metwally, "Google Search Trend of Dengue fever in developing Countries in 20132014: An Internet-Based Analysis", Journal of Health Informatics in Developing Countries, Vol. 9, No. 1, 2015.

[25]R. Jain, S. Sontisirikit, S. Iamsirithaworn, and H. Prendinger, "Prediction of dengue outbreaks based on disease surveillance, meteorological and socio-economic data", BMC Infectious Diseases, Vol. 19, No. 1, p. 272, 2019.

[26]Z. Husnina, A. C. A. Clements, and K. Wangdi, "Forest cover and climate as potential drivers for dengue fever in Sumatra and Kalimantan 20062016: a spatiotemporal analysis", Tropical Medicine and International Health, Vol. 24, No. 7, pp. 888-898, 2019.

[27]A. Appice, Y. R. Gel, I. Iliev, V. Lyubchich, and D. Malerba, "A Multi-Stage Machine Learning Approach to Predict Dengue Incidence: A Case Study in Mexico", IEEE Access, Vol. 8, pp. 52713-52725, 2020.

[28]M. Salathé, "Digital epidemiology: what is it, and where is it going?", Life Sciences, Society and Policy, Vol. 14, No. 1, p. 1, 2018.

[29] Y. Zhang, H. Bambrick, K. Mengersen, S. Tong, L. Feng, L. Zhang, G. Liu, A. Xu, and W. Hu, "Using big data to predict pertussis infections in Jinan city, China: a time series analysis", International Journal of Biometeorology, Vol. 64, No. 1, pp. 95-104, 2020.

[30] Y. Zhang, L. Yakob, M. B. Bonsall, and W. Hu, "Predicting seasonal influenza epidemics using cross-hemisphere influenza surveillance data and local internet query data", Scientific Reports, Vol. 9, 2019.

[31]"BULETINAPJIIEDISI74November2020.pdf." Accessed: Mar. 31, 2021. [Online]. Available: https://apjii.or.id/downfile/file/BULETINAPJII EDISI74November2020.pdf

[32] A. S. Jati, "Jumlah Pengguna Twitter Meningkat, Tapi... [The number of Twitter users is increasing, but ...]", detikinet. https://inet.detik.com/cyberlife/d-

5001786/jumlah-pengguna-twitter-meningkattapi (accessed Feb. 27, 2021).

[33]P. KOMINFO, "Indonesia Peringkat Lima Pengguna Twitter [Indonesia Ranks Five Twitter Users]", Website Resmi Kementerian Komunikasi dan Informatika RI. http:///content/detail/2366/\%20indonesiaperingkat-limapenggunatwitter/0/sorotan_media (accessed Feb. 27, 2021).

[34]S. Mala and M. K. Jat, "Implications of meteorological and physiographical parameters on dengue fever occurrences in Delhi”, Science of the Total Environment, Vol. 650, pp. 22672283, 2019.

[35]A. Q. Munir, S. Hartati, and A. Musdholifah, "Early Identification Model for Dengue Haemorrhagic Fever (DHF) Outbreak Areas Using Rule-Based Stratification Approach", International Journal of Intelligent Engineering \& Systems, p. 15, 2018.

[36]S. Jiang, R. Xiao, L. Wang, X. Luo, C. Huang, J. Wang, K. Chin, and X. Nie, "Combining Deep Neural Networks and Classical Time Series Regression Models for Forecasting Patient Flows in Hong Kong”, IEEE Access, Vol. 7, pp. 118965-118974, 2019.

[37]J. Xu, K. Xu, Z. Li, F. Meng, T. Tu, L. Xu, and Q. Liu, "Forecast of Dengue Cases in 20 Chinese Cities Based on the Deep Learning Method", International Journal of Environmental Research and Public Health, Vol. 17, No. 2, 2020.

[38] K. Lan, D. Wang, S. Fong, L. Liu, K. K. L. Wong, and N. Dey, "A Survey of Data Mining and Deep Learning in Bioinformatics", Journal of Medical Systems, Vol. 42, No. 8, p. 139, 2018.

[39]M. I. Jordan and T. M. Mitchell, "Machine learning: Trends, perspectives, and prospects", Science, Vol. 349, No. 6245, pp. 255-260, 2015.

[40]S. Chae, S. Kwon, and D. Lee, "Predicting Infectious Disease Using Deep Learning and Big Data", International Journal of Environmental Research and Public Health, Vol. 15, No. 8, p. 1596, 2018.

[41]C. Bergmeir and J. M. Benítez, "On the use of cross-validation for time series predictor evaluation”, Information Sciences, Vol. 191, pp. 192-213, 2012.

[42]"Pemerintah Kota Surabaya." https://www.surabaya.go.id/id/page/0/8227/geo grafi (accessed Mar. 31, 2021). 
[43]"Dinas Komunikasi dan Informatika Kota Surabaya [Surabaya City Government]." https://dinkominfo.surabaya.go.id/index.php?pa ges=detail_berita\&id_berita $=190$ (accessed Mar. 31, 2021).

[44]"malangkab-Kondisi Geografis.pdf [Geographical Condition of Malang District]." Accessed: Mar. 05, 2021. [Online]. Available: http://malangkab.go.id/uploads/dokumen/malan gkab-Kondisi\%20Geografis.pdf

[45] G. Cervellin, I. Comelli, and G. Lippi, "Is Google Trends a reliable tool for digital epidemiology? Insights from different clinical settings", Journal of Epidemiology and Global Health, Vol. 7, No. 3, pp. 185-189, 2017. 